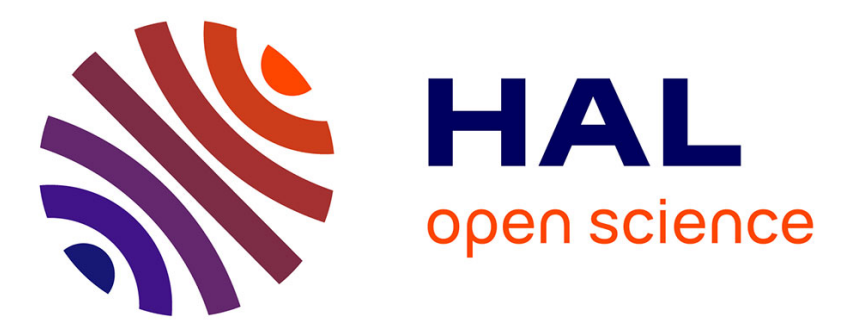

\title{
The RF power coupler development programme at LAL-Orsay and DESY-Hamburg for TESLA and the European X-FEL
}

\author{
T. Garvey, W.-D. Möller
}

\section{- To cite this version:}

T. Garvey, W.-D. Möller. The RF power coupler development programme at LAL-Orsay and DESY-Hamburg for TESLA and the European X-FEL. International Congress on Optics and OptoElectronics, Aug 2005, Warsaw, Poland. 594803 (13 p.), 10.1117/12.623784 . in2p3-00024602

HAL Id: in2p3-00024602

https://hal.in2p3.fr/in2p3-00024602

Submitted on 26 Sep 2005

HAL is a multi-disciplinary open access archive for the deposit and dissemination of scientific research documents, whether they are published or not. The documents may come from teaching and research institutions in France or abroad, or from public or private research centers.
L'archive ouverte pluridisciplinaire HAL, est destinée au dépôt et à la diffusion de documents scientifiques de niveau recherche, publiés ou non, émanant des établissements d'enseignement et de recherche français ou étrangers, des laboratoires publics ou privés. 


\title{
The RF power coupler development programme at LAL-Orsay and DESY-Hamburg for TESLA and the European X-FEL
}

\author{
T. Garvey*a and W.-D. Möller ${ }^{\mathrm{b}}$ \\ ${ }^{a}$ Laboratoire de l'Accélérateur Linéaire, IN2P3-CNRS, Université de Paris-Sud, \\ B.P. 34, 91898 Orsay, France ; \\ ${ }^{\mathrm{b}}$ Deutsches Elektronen-Synchrotron, Notkestrasse 85, D-22607 Hamburg, Germany.
}

\begin{abstract}
In the context of a collaboration between LAL (Orsay) and DESY (Hamburg) a programme of development and tests of proto-type power couplers for superconducting cavities is underway in Orsay. Such couplers need to be developed for linear accelerators which require high gradient superconducting cavities, such as the European X ray Free Electron Laser or the International Linear Collider (ILC) project. We will describe the technical demands which have to be met to build such couplers and will present proto-type designs which are intended to meet these demands, taking the ILC as an example. A description of the infra-structure necessary for the coupler development will also be given along with first high power tests results on a series of power couplers built in industry.
\end{abstract}

Keywords: power couplers, superconducting RF, linear collider, X-ray FEL

\section{INTRODUCTION}

Recent years have seen an increasing interest in the use of radio-frequency (RF) linear accelerators (linacs) using superconducting (SC) cavities. These linacs are being built, or are under consideration, for diverse applications such as the production of neutrons by spallation, high energy linear colliders, short wavelength free electron lasers (FEL), making use of the SASE (Self-Amplified Spontaneous Emission) mechanism, and as Energy Recovery Linacs (ERL) for synchrotron radiation sources. In February of 2003 the German government announced its approval for the construction, at DESY, of a $20 \mathrm{GeV} \mathrm{SC} \mathrm{linac} \mathrm{as} \mathrm{a} \mathrm{driver} \mathrm{for} \mathrm{a} \mathrm{SASE} \mathrm{type} \mathrm{X-ray} \mathrm{FEL,} \mathrm{on} \mathrm{condition} \mathrm{that} \mathrm{it} \mathrm{can} \mathrm{be} \mathrm{built}$ with European co-operation. In January of 2004 the International Committee for Future Accelerators (ICFA) established a panel of experts to recommend the technology of choice for the construction of a linear collider in the TeV energy range. In August 2004 this panel, the International Technology Recommendation Panel (ITRP), endorsed superconducting RF (SRF) technology for the construction of the International Linear Collider (ILC). The enthusiasm for SRF today is largely due to the spectacular progress made in this field by the DESY laboratory and its collaborators within the international collaboration, TESLA (Tera Electron-volt Superconducting Linear Accelerator). Record accelerating gradients $(>35 \mathrm{MV} / \mathrm{m})$ with high quality factors $\left(>1 \times 10^{10}\right)$ have been achieved with multi-cell cavities at DESY making SRF an attractive option for many new projects. Nevertheless, if the enormous progress made in the production of high gradient SC cavities is to be fully exploited in operational accelerators it is essential that all ancillary components meet the required performances. Among the most critical of these components is the input power coupler which is used to excite the accelerating mode of the cavity. We will discuss the program of work which is in progress at DESY and at Orsay for the development of couplers for the TESLA Test Facility VUV-FEL, the planned X-FEL as well as the development of proto-types which may be of interest for the ILC.

\section{THE TTF-III COUPLER}

\subsection{The coupler function}

The principle function of the coupler is to transfer power from the RF source (typically a klystron) to that place where the power is used to accelerate the electron beam (the superconducting RF cavity). Thus, the coupler is essentially no more than a length of wave-guide which should transport power to the cavity with the minimum of reflection or transmission loss. However, the design of the coupler is further complicated by the fact that it must fulfil the following 
additional functions; (i) the coupler must play the role of a vacuum barrier between the air-filled wave-guide and the accelerating cavity, (ii) the coupler must minimise the thermal losses between the feed waveguide, at room temperature, and the RF cavity, which is at $2.0 \mathrm{~K}$ in the case of TTF.

Before describing the coupler it is worthwhile to describe briefly the basic cavity and cryo-module arrangement used on TTF. The TTF cavities are standing wave resonators operated in $\pi$ mode at a frequency of $1.3 \mathrm{GHz}$. They are composed of 9 cells each one half wavelength long as they are designed for ultra-relativistic beams. The TTF cryo-modules are equipped with eight such cavities, each one powered by an individual coupler. A detailed discussion of the TESLA cavity and cryo-module can be found in references 1 and 2 respectively.

\subsection{Description of the TTF-III coupler}

The TESLA Test Facility (TTF) linac was built as a proto-type of the TESLA collider. Several variants of coupler have been used on this linac. Their characteristics can be found elsewhere ${ }^{3,4,5}$. Here we will concentrate on the coupler which has essentially been chosen for use with the European X-FEL, the so-called TTF-III coupler. A schematic illustration of the TTF-III coupler is shown in figure 1.

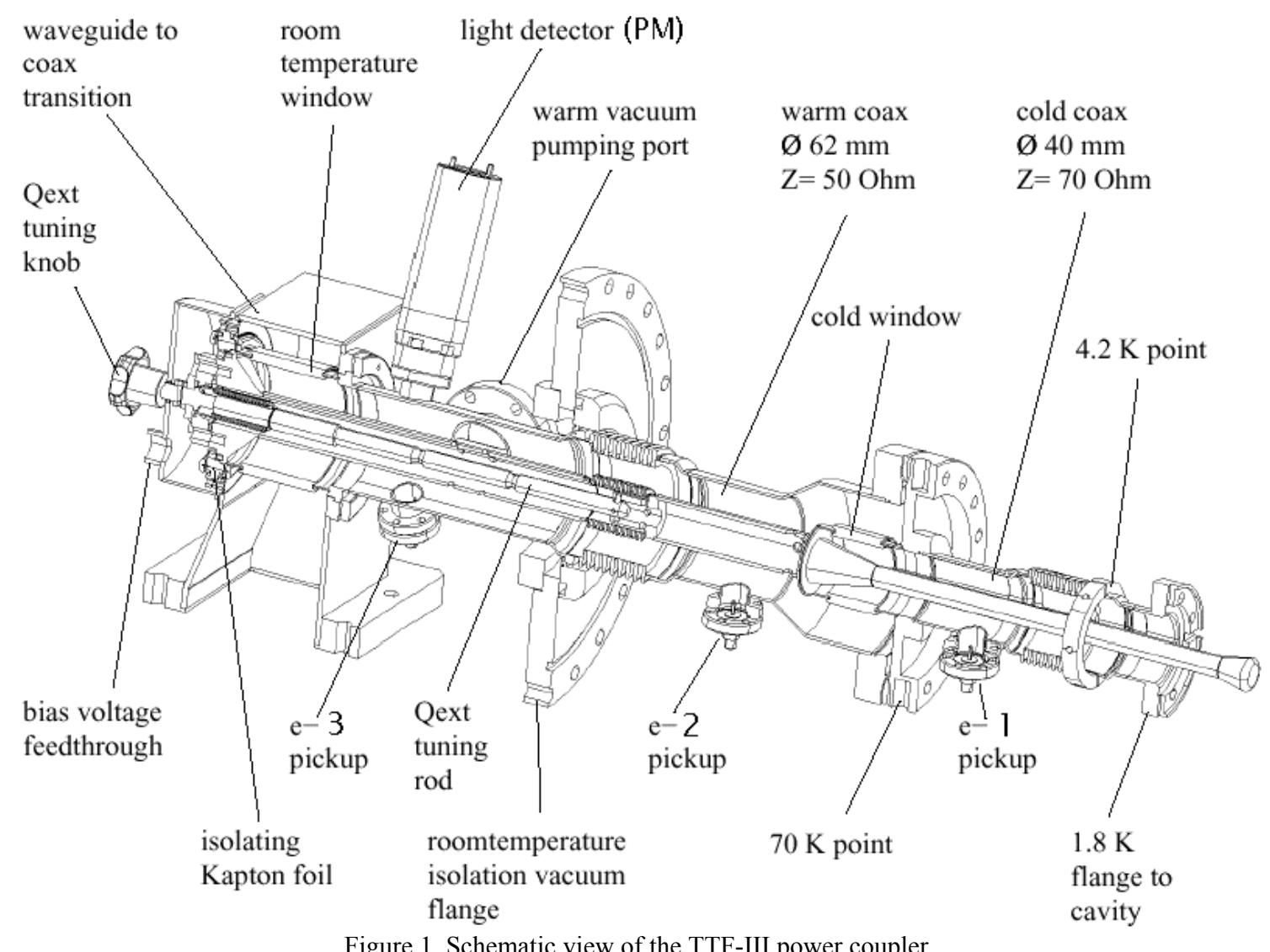

The coupler design is essentially a co-axial line. Coupling to the cavity is made capacitively through the inner conductor, or antenna. Broadly speaking it can be divided into three main parts:

(i) The "cold" assembly. This is a $70 \Omega$ line of $40 \mathrm{~mm}$ outside diameter (o.d.). The cold assembly includes a dielectric window, which maintains the cavity vacuum and also keeps the coupler free from dust and other contaminants, which would otherwise limit the performance of the cavity. The antenna is rigidly connected to this window.

(ii) The "warm" coupler assembly. This is a $50 \Omega$ line of $62 \mathrm{~mm}$ o.d. It incorporates a "warm" ceramic dielectric window, which allows the co-axial line to be maintained under vacuum. 
A half-height waveguide to co-axial transition. This transition matches the impedance of the rectangular waveguide coming from the klystron to the co-axial coupler line.

The ceramic windows are manufactured from alumina, $\mathrm{Al}_{2} \mathrm{O}_{3}$, with $97.6 \%$ purity. The vacuum sides of the ceramic surfaces have a $\sim 10 \mathrm{~nm}$ deposition of titanium-nitride. This is done in order to reduce the secondary electron emission coefficient of the ceramic, so as to minimise problems with multipactoring (see below). In order to vary the strength of the coupling to the cavity the antenna can be moved axially by adjusting a threaded screw positioned at the extremity of the air side of the coupler. This adjustment is possible through the use of bellows in the cold outer conductor and the warm inner and outer conductors. A high voltage feed-through allows one to apply a D.C. bias to the central conductor relative to the grounded outer conductor. This is done to further combat problems with multipactoring. A Kapton foil provides the insulation between the conductors. A number of diagnostics are deployed on the couplers. There are feedthroughs for electron pick-ups in both the warm and cold parts. A photo-multiplier is also employed on the vacuum side of the room-temperature window. There are also ports in the air side of the wave-guide transition equipped with an arc detector and an infra-red detector for measurement of the ceramic temperature. With the exception of the antenna, which is made of copper, the coupler assemblies are machined from stainless steel. The poor thermal conductivity of steel helps to reduce heat flux from room temperature towards the inside of the cryostat. However, to reduce RF losses in the coupler, the RF surfaces are coated with a few skin-depths of copper (typically $\sim 30 \mu \mathrm{m}$ for the inner conductor and 10 $\mu \mathrm{m}$ for the outer conductor).

In the original TESLA design, the cavities were intended to accelerate an $8 \mathrm{~mA}$ beam at a gradient of $25 \mathrm{MV} / \mathrm{m}$ for a pulse of $800 \mu$ s duration and $\sim 500 \mu$ s cavity filling time at a repetition rate of $10 \mathrm{~Hz}$. Thus the peak and average power requirements were $208 \mathrm{~kW}$ and $2.8 \mathrm{~kW}$ respectively. The parameters of the TESLA proposal have varied over the years and the TESLA Technical Design Report includes parameter sets for $500 \mathrm{GeV}$ and $800 \mathrm{GeV}$ centre of mass colliders. The coupler power requirements for these machines are shown in table 1. The higher power for the $800 \mathrm{GeV}$ option is due to the assumption that a single coupler would power two 9 cell cavities. The "superstructure" column refers to a proposed cavity composed of four 7-cell resonators. The X-FEL project will use the standard 9 cell cavity and the coupler requirements are shown in table 1 for comparison. In practice one should include an additional $\sim 25 \%$ power in order to include RF control for frequency de-tuning. The needs of the ILC can be taken to be similar to those of TESLA$800 \mathrm{GeV}$. Note that the TESLA machine would require more than 20,000 couplers.

\begin{tabular}{|l|c|c|c|c|c|}
\hline & TTF & TESLA 500 & TESLA 800 & Superstructure & X-FEL \\
\hline Beam power & $208 \mathrm{~kW}$ & $230 \mathrm{~kW}$ & $925 \mathrm{~kW}$ & $1440 \mathrm{~kW}$ & $200 \mathrm{~kW}$ \\
& & & & & \\
\hline Repetition rate / & $10 \mathrm{~Hz}$ & $5 \mathrm{~Hz}$ & $4 \mathrm{~Hz}$ & $4 \mathrm{~Hz}$ & $10 \mathrm{~Hz}$ \\
Pulse length & $1.33 \mathrm{~ms}$ & $1.37 \mathrm{~ms}$ & $1.33 \mathrm{~ms}$ & $1.33 \mathrm{~ms}$ & $1.33 \mathrm{~ms}$ \\
\hline coupling & $10^{6}-10^{7}$ & $3 \times 10^{6}$ & $2.5 \times 10^{6}$ & $2.5 \times 10^{6}$ & $10^{6}-10^{7}$ \\
\hline
\end{tabular}

Table 1. Power requirements of different couplers.

Of course there are also requirements on the cryogenic performance of the couplers (static and dynamic losses). These are not shown in table 1 as this paper will essentially be concerned with room temperature power tests. However, it is worth noting that experience with TTF shows that the cryogenic performance is achieved using the TTF-III coupler.

\subsection{Coupler manufacture}

It should be clear from the description given above that several technologies are necessary for the manufacture of the couplers. Precision welding (by electron beam, TIG, laser), vacuum brazing of ceramic to metal, high quality copper coating of smooth/bellowed surfaces and thin film evaporation are all required. In addition, as these are UHV components which must not degrade the cavity performance, it is essential to use ultra-clean handling procedures at each stage. For this reason, it is desirable that couplers be manufactured by companies having the relevant skills and experience. Until recently, TTF-III couplers were manufactured in relatively small numbers as and when required. In summer of 2002, the largest single order to date for TTF-III couplers was placed with the company CPI (Boston, USA) for the future modules of the TTF VUV-FEL. CPI was asked to produce thirty couplers which have since been delivered to Orsay and are presently under test. The tests of the first TTF-III couplers have been performed at DESY and have been described in reference 6 . Here we will concentrate on the tests of the couplers manufactured by CPI. Figure 2 shows various coupler parts produced by CPI. 


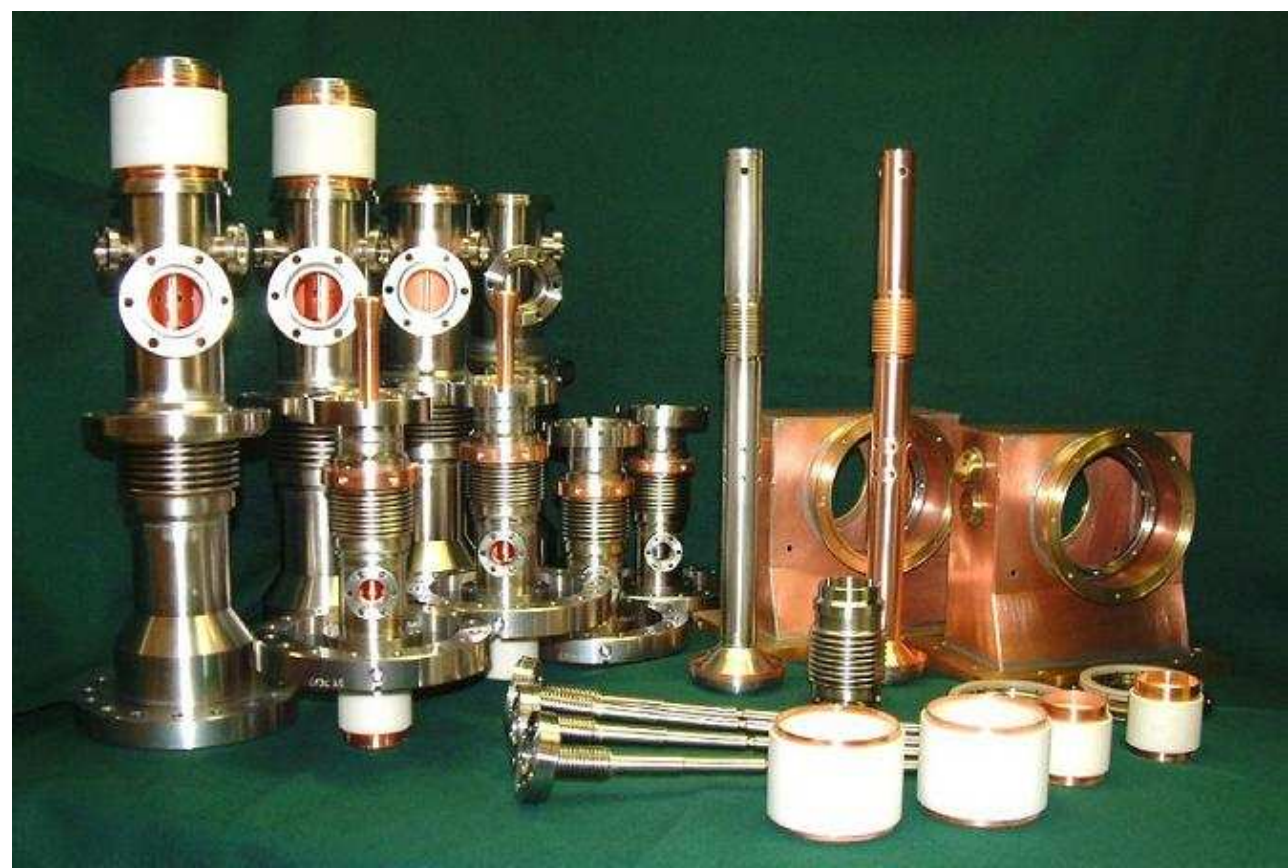

Figure 2. Various coupler parts including warm and cold assemblies, ceramic windows, wave-guide transitions and bellows.

\subsection{Infra-structure for preparation and tests of couplers}

As noted above, the cold assembly shares a common vacuum with the cavity. For this reason this assembly has to be prepared with all the care and attention given to the superconducting niobium resonators. After arrival at Orsay the couplers are inspected for dimensional conformity (where possible) and their general visual aspect. An endoscopic inspection bench, coupled to a CCD camera, has been built to observe and record the quality of the internal welds, braze joints, and surfaces (Fig. 3). Each coupler assembly is marked for trace-ability and it is hoped that couplers, which show unusual behaviour during RF tests, might be correlated with any apparent manufacturing anomalies. After inspection the couplers are stored in cabinets under dry nitrogen while awaiting tests.

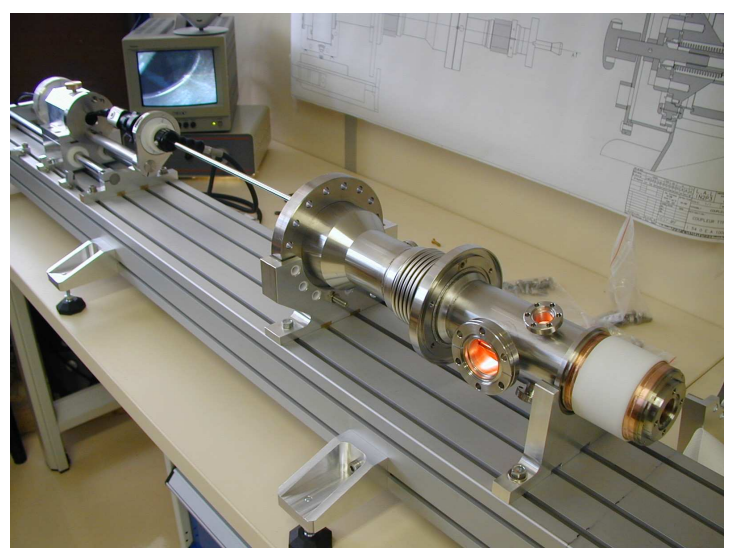

Figure 3. Power coupler inspection bench

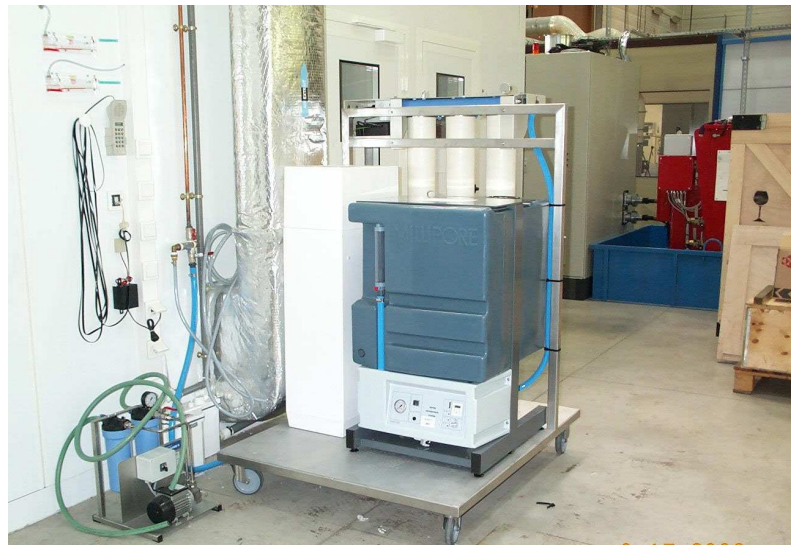

Figure 4. Ultra-pure water production system.

The coupler parts are first cleaned using ultra-pure (UP) water (electrical resistivity of $18 \mathrm{M} \Omega \mathrm{cm}$.) A commercial system for UP water production has been installed for this purpose (Fig. 4). The cleaning in UP water takes place in an ultra-sonic bath with a solution of detergent. After cleaning, the coupler parts are rinsed, again with UP water and then left to dry under the laminar flow of a class 10 clean room. The couplers are then baked out in a custom built vacuum furnace (base pressure with load $-10^{-6} \mathrm{mbar}$ ) at $400^{\circ} \mathrm{C}$ for four hours. This bake-out serves to out-gas adsorbed hydrogen, water and organic compounds. The cold coupler parts are then mounted, in pairs, onto a small non-standard 
wave-guide test box in a class 10 clean room (Fig. 5). The vacuum oven has the attractive feature that it is contiguous with the class 10 clean room so that couplers can exit the oven straight into the clean environment (Fig. 6).

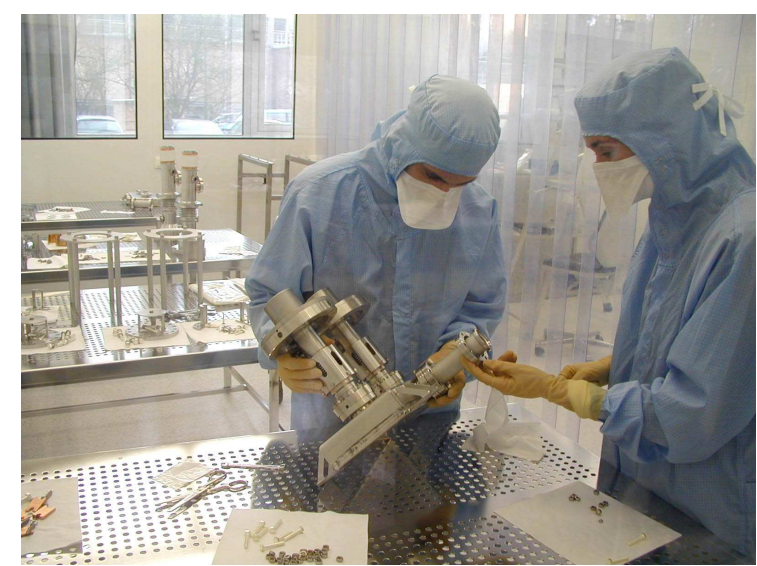

Figure 5. Assembly of cold coupler parts in class 10 clean room

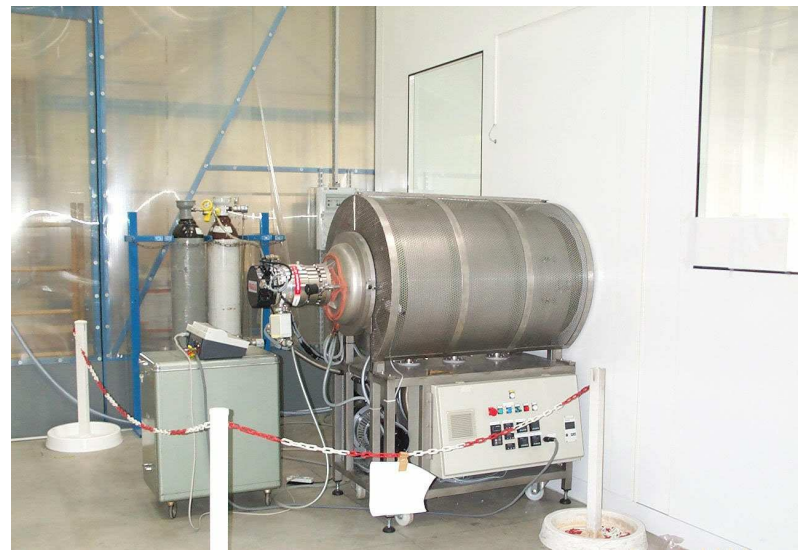

Figure 6. Vacuum oven for coupler bake-out

Once checked to verify they are vacuum tight, the couplers are then installed on the high power test bench. The waveguide transitions are installed under a laminar flow equivalent to class 100.

\section{HIGH POWER RF TESTS}

\subsection{Conditioning}

"Conditioning" is that process whereby the couplers are gradually brought from zero power up to their operational peak and average power levels. When couplers see RF power for the first time it is typical to observe dramatic increases in vacuum pressure usually accompanied by the detection of electron signals within the coupler. Two types of electrical discharge can be responsible for these signals. The first of these, multipactoring, is a ubiquitous phenomena whenever one has RF power in a vacuum environment ${ }^{7}$. It is due to a resonant electron discharge which can rapidly build up if the geometrical conditions and RF field amplitude are favourable at the operating frequency. However, in order to be sustained, the secondary electron emission (SEE) coefficients, $\delta$, of the materials involved must be greater than unity. In this case an electron impacting the surface will produce $\delta$ electrons and these $\delta$ electrons will then produce $\delta^{2}$ and so on giving rise to an avalanche discharge. The energy deposited by these multipacting electrons can be damaging for the ceramic windows and so it is particularly important to avoid multipactor in the region of the windows. The SEE of alumina ceramics is known to be well above 1 (typically $3.5-7$ ) and the reason for the titanium nitride coating on the ceramic is to reduce this value ${ }^{8}$. The SEE coefficients of the materials from which the coupler is fabricated are strongly dependent upon their surface conditions. The presence of adsorbed gases on the surfaces of the copper coatings and ceramics can radically change the values from those of the 'pure' surface state. The effect of the electron impacts on the RF surfaces is thought to desorb these gases thus changing (reducing) the SEE values of the surface. In this sense, the multipacting contributes to the conditioning of the coupler. However, it is desirable to prevent the build up the electron density so as to avoid an electrical spark which could result in serious damage to the coupler. As the discharge stops at the end of the RF pulse, the desorbed gases can be removed by the pumping system during the time between RF pulses, if it is sufficiently fast. Therefore, one can consider that the conditioning procedure essentially consists of "cleaning" the vacuum surfaces in a controlled and safe manner.

The second source of electrons is field emission. Although the calculated RF electrical fields in the coupler are too low to cause breakdown in vacuum $(<1 \mathrm{MV} / \mathrm{m})$ it is likely that the couplers have a number of 'microscopic' imperfections on the inner metallic surfaces. It would be sufficient for a small braze or weld default to leave sharp points inside the coaxial line thus significantly enhancing the local electric field over the average value. If the copper coatings are not sufficiently smooth (problems are frequently found with adhesion of the coating to the stainless steel surface) then metallic "whiskers" may be present which will also produce high peak fields. The field emission current produced from these points and whiskers is believed to "burn off" the emitters resulting in their removal. 


\subsection{The RF power source}

The couplers are tested and conditioned in pairs. The high power RF source is a 5 MW THALES klystron (TH 2104C) and a pulsed modulator of the type developed for $\mathrm{TTF}^{2}$. The wave-guide distribution system includes directional WG couplers for the measurement of incident, reflected and transmitted power. A four port differential phase-shift circulator protects the klystron in case of excessive reflected power. A schematic of the test arrangement is shown in Fig. 7 and a picture of two couplers under test can be seen in Fig. 8 .

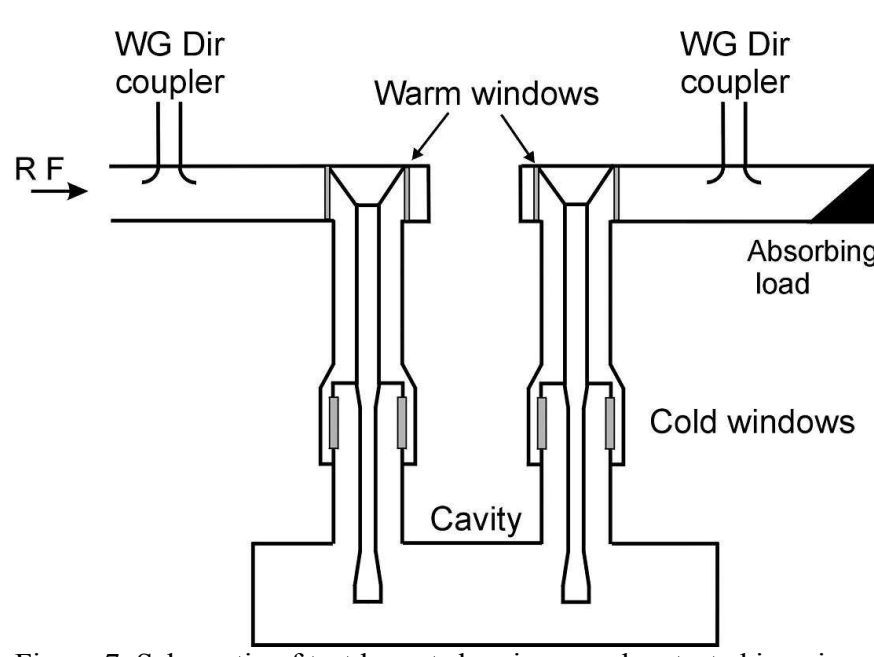

Figure 7. Schematic of test layout showing couplers tested in pairs.

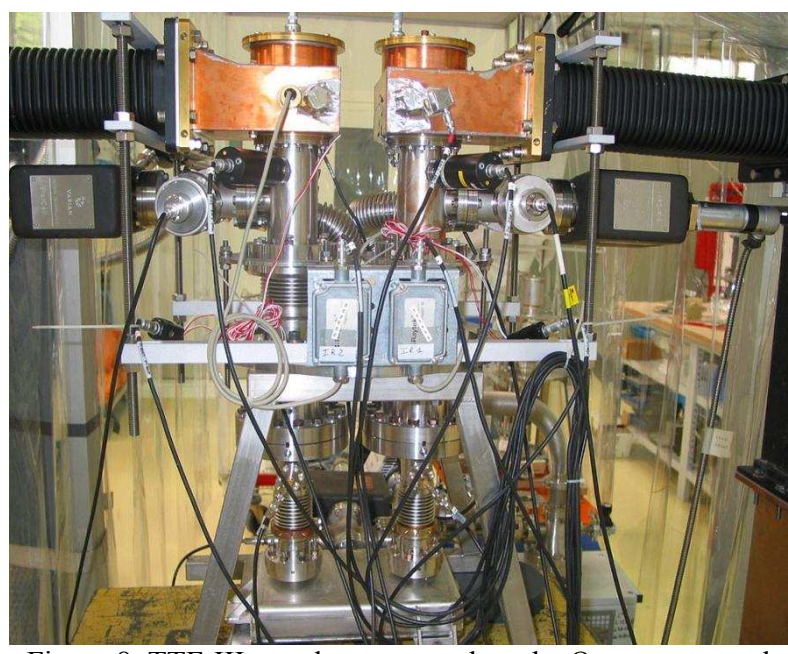

Figure 8. TTF-III couplers mounted on the Orsay test stand.

As the couplers are used in pulse mode of operation, there are standing waves during the transient filling of the cavity. In this case the RF voltages can be double the value used in travelling wave mode. Hence it is prudent to test the couplers up to power levels a factor of 4 greater than the nominal power. The TTF-III couplers are tested to $1 \mathrm{MW}$.

\subsection{Conditioning procedure}

After the couplers are mounted onto the RF test stand we apply an additional in-situ bake-out. This bake-out is limited to $130^{\circ} \mathrm{C}$ by an aluminium seal in the cold coupler flange. This procedure requires several days, including the time to install the heating tapes and temperature surveying equipment, the gradual increase in heating temperature, the actual bake-out period (24 hours) and then the cool down period. On the other hand, there is a significant reduction in the required RF conditioning time due to this bake-out. The test stand is equipped with turbo-molecular pumps to provide a rough vacuum and 20 litre/second ion pumps mounted on each warm assembly and on the test box connecting the two cold assemblies. After the in-situ bake-out we achieve a base pressure of $2 \times 10^{-9} \mathrm{mbar}$. The gradual increase of power is carried out in the following fashion. The RF pulse length is first set to $20 \mu$ s the and power increased in steps of $0.1 \mathrm{~dB}$, every thirty seconds, from $1 \mathrm{~kW}$ to $1 \mathrm{MW}$. The pulse repetition frequency is $2 \mathrm{~Hz}$. The increment in power is only permitted on condition that the vacuum level stays below a specific value $\left(2 \times 10^{-7} \mathrm{mbar}\right)$. Light levels measured by the photo-multiplier and electron levels are also used as interlocks. If the vacuum level exceeds $10^{-6}$ mbar the power is stopped until the vacuum recovers and then the procedure is re-started. After the $1 \mathrm{MW}$ level is obtained the power level is reset to $1 \mathrm{~kW}$ and the pulse width increased to $50 \mu \mathrm{s}$. The power is then increased to $1 \mathrm{MW}$ in the same fashion. This procedure is then repeated for pulse widths of 100, 200 and $400 \mu \mathrm{s}$. The power ramp is then repeated for pulses of 800 and $1300 \mu \mathrm{s}$ but to a peak power of only $500 \mathrm{~kW}$.

The control and data acquisition of the conditioning is performed using an industrial PC and LabView programming ${ }^{9}$. This system controls the application of the high power pulses including synchronisation between modulator and klystron as well as the acquisition of data from all diagnostics, using analogue to digital converters (National Instruments PCI$6071 \mathrm{E})$. It also allows control of the vacuum system and surveys the status of interlocks to allow the system to decide on which action to take on the next pulse. 


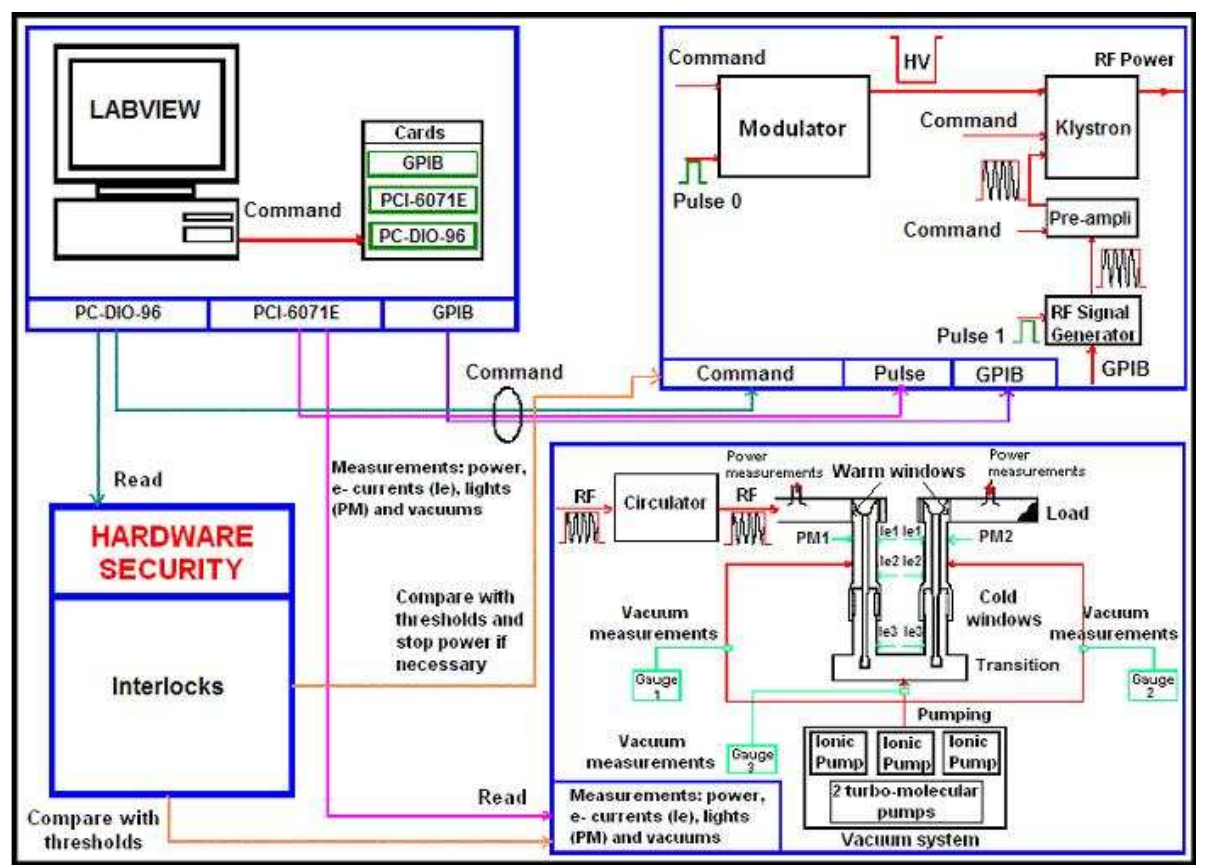

Figure 9. Schematic of the control and data acquisition system for coupler conditioning.

\subsection{Results and discussion}

A typical variation of power with time during a conditioning run is shown in Fig. 10. Note that the time needed to reach $1 \mathrm{MW}$ of power is clearly longest for the first, $20 \mu \mathrm{s}$, ramp. Although this is the shortest pulse, it is also the first time that the coupler is submitted to RF power and so the time needed to reach $1 \mathrm{MW}$ can be understood in terms of gas desorption. The measured electron signals for different pulse widths are shown as a function of power in Fig. 11. One sees clearly that the power level at which electrons are detected increases for each successive ramp in pulse-width. For the $1300 \mu$ s sweep there is essentially no further electron activity even at the highest power level.

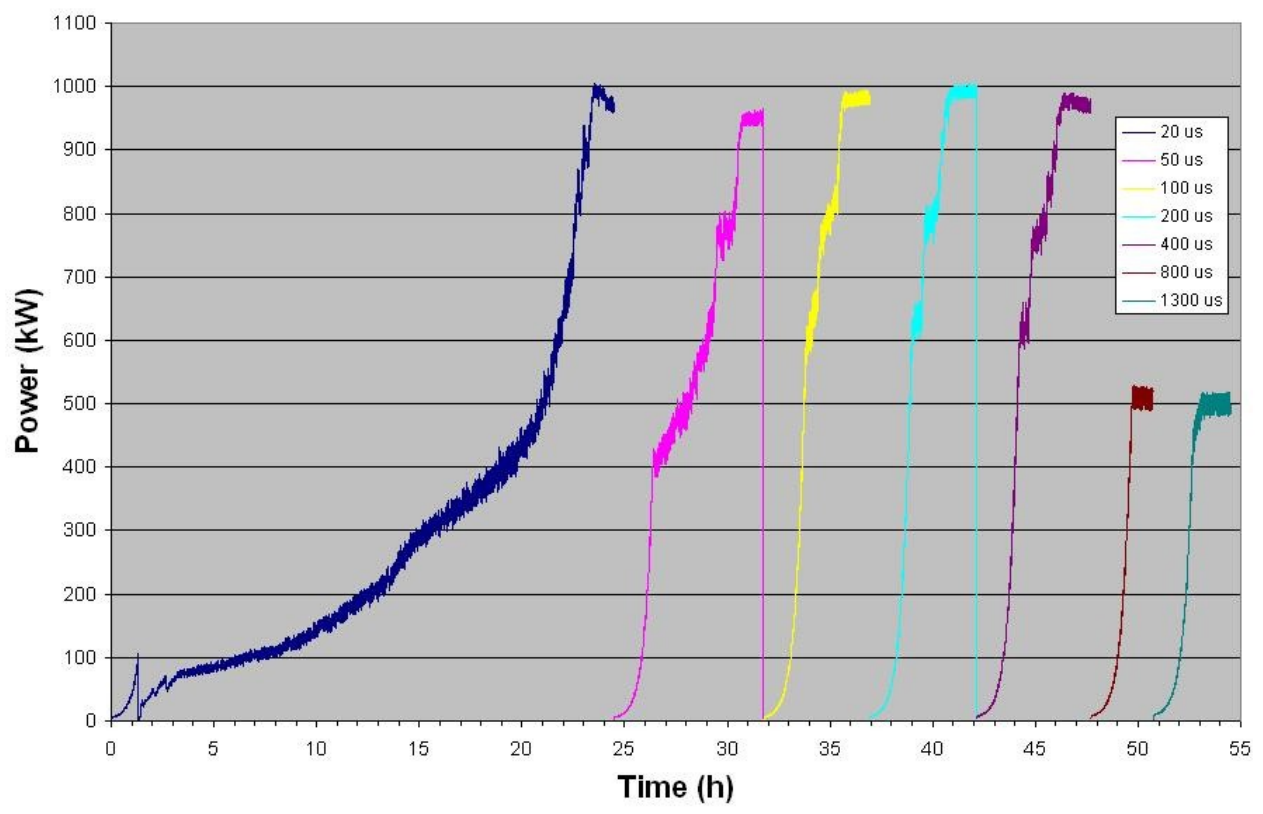

Figure 10. Typical RF power ramp obtained during conditioning. 
At the time of writing, seven pairs of TTF-III couplers have been conditioned at Orsay. The measured conditioning times are shown in the form of a histogram in Fig. 13. The benefits of the in-situ baking are clearly apparent. However, even for the baked couplers one notes a variation of between 44 and 72 hours for conditioning. These data are similar to the experience found at DESY, with a larger data set, where conditioning on a test stand with in-situ baking showed conditioning times varying from $\sim 60$ to 140 hours ${ }^{6}$. The reason for the large spread in the conditioning time for different couplers is not understood. One needs to identify what differences between couplers could be responsible for this spread. Variations resulting from their manufacture may be one reason. Alternatively, differences in preparation, handling and/or mounting prior to tests may also be responsible for the spread. This question, along with the quest for procedures to further reduce the conditioning times will be one of our principal lines of future investigation.

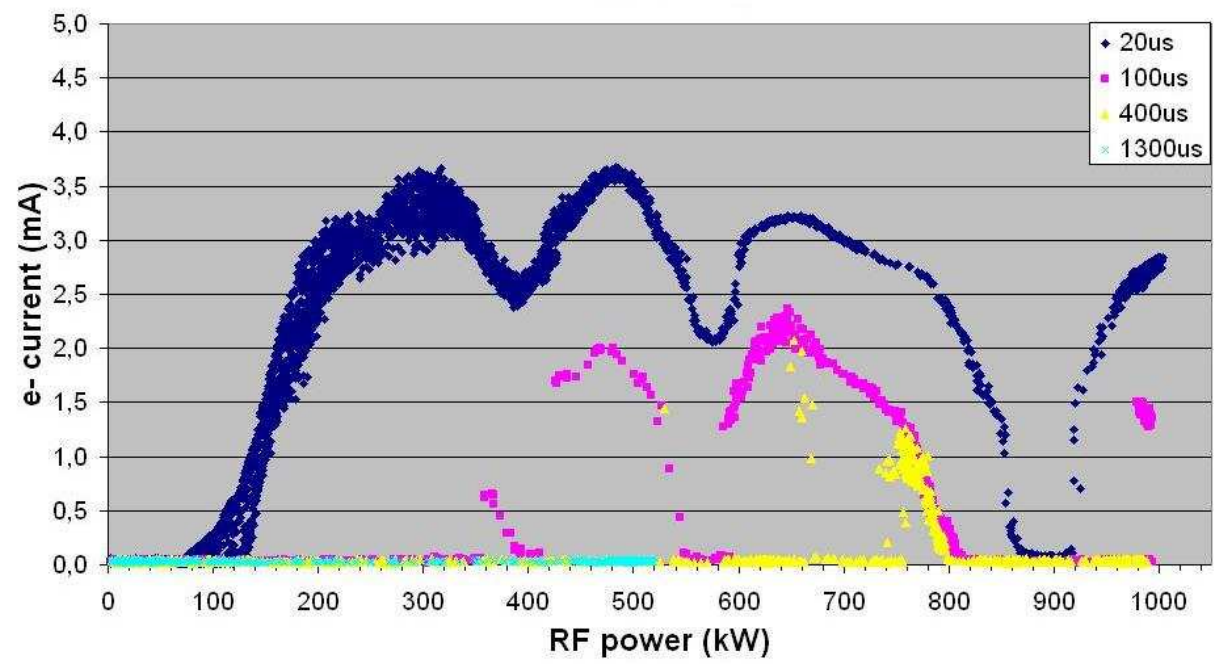

Figure 11. Measured electron current as a function of RF power for different pulse widths.

A typical electron signal as a function of power and pulse-width is shown in Fig. 11. The electrons make their first appearance at power levels of $80-100 \mathrm{~kW}$. Although they are present throughout the ramp they are below the level we consider to be dangerous ( $5 \mathrm{~mA}$ ). After the first ramp to $1 \mathrm{MW}$, subsequent ramps at larger pulse-widths indicate greatly reduced electron signals, illustrating the conditioning action. The signal is essentially zero or, at worst, very low for the $1300 \mu \mathrm{s}$ ramp. Fig. 12 shows the spectra of residual gases before bake-out and at the end of the conditioning. Prior to the bake-out the spectrum is dominated by hydrogen, water vapour, nitrogen and oxides of carbon. At the end of conditioning, the dominant feature is hydrogen.

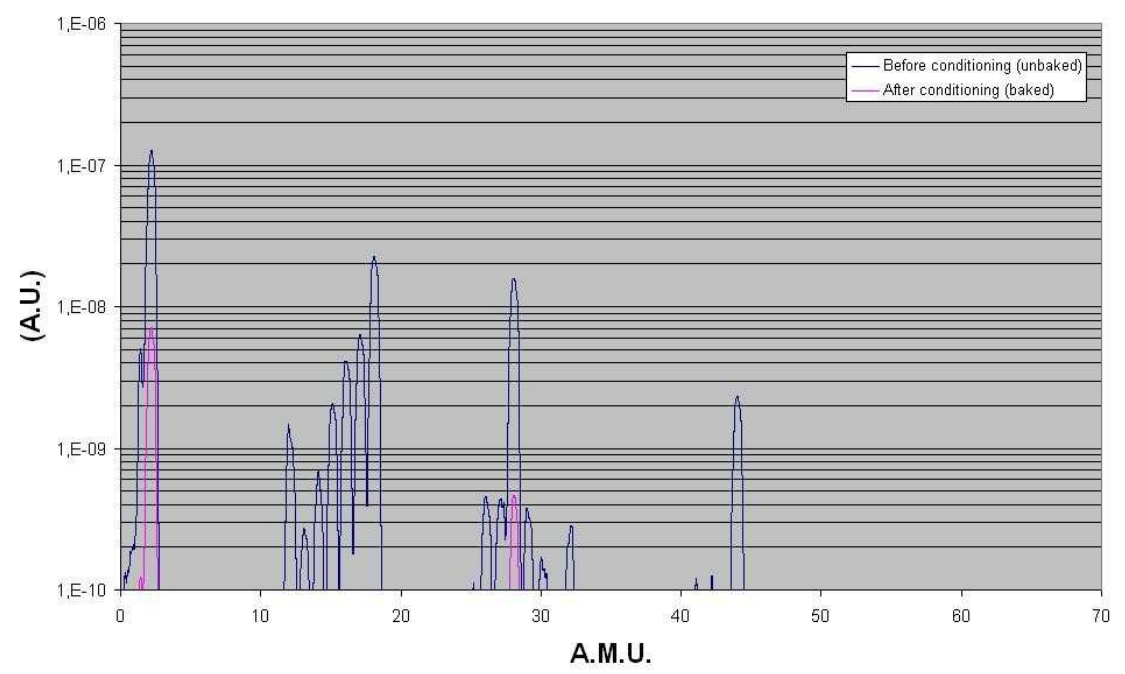

Figure 12. Residual gas analyser plots showing the partial pressures levels (in arbitrary units) as a function of atomic mass. The blue plot shows the pressure prior to baking and conditioning. The red plot shows the pressure after baking and conditioning. 


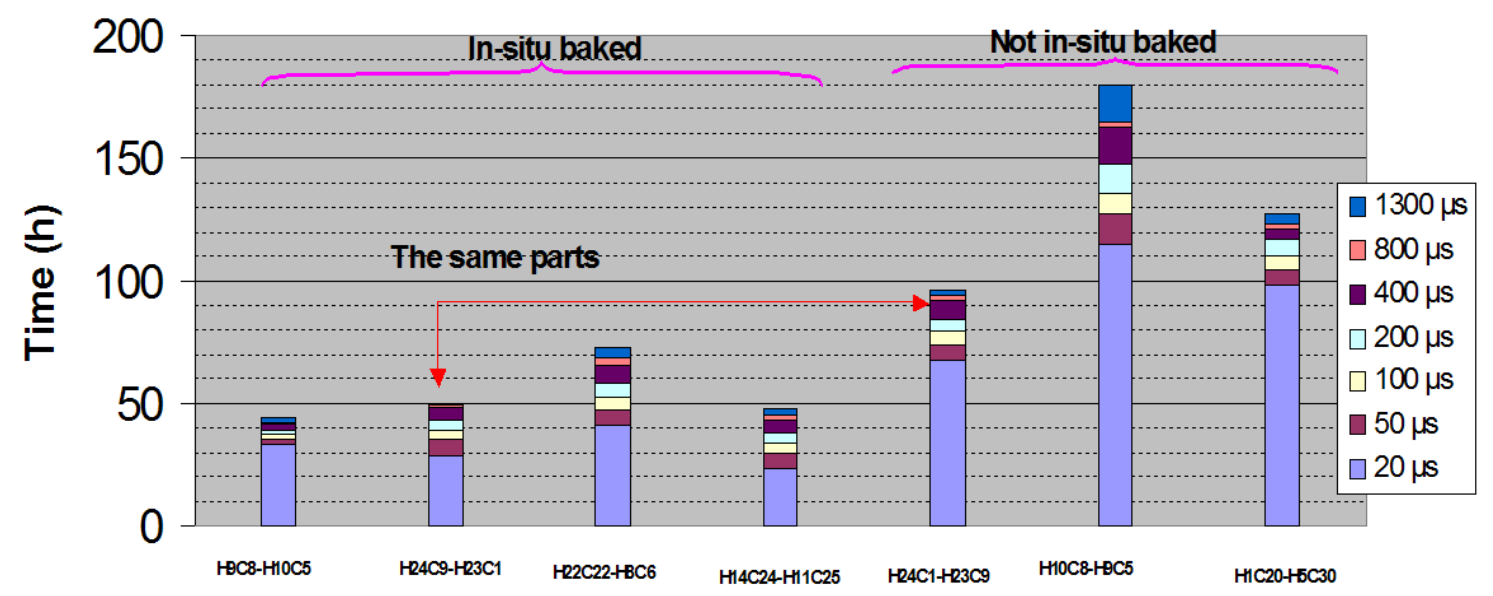

Figure 13. Histogram of conditioning times for seven coupler pairs illustrating the difference between baked and un-baked couplers.

At DESY the power couplers are also conditioned individually on super-conducting cavities within a horizontal test cryostat and then, finally, in groups of 8 after being mounted to the super-conducting modules. In this case the performance of the coupler can be measured in its low temperature operating environment. It is worth noting that TTFIII couplers have been tested with an electro-polished cavity at $35 \mathrm{MV} / \mathrm{m}$. The applied power was $600 \mathrm{~kW}$ at this gradient due to the fact that the RF feedback system was not in operation properly. A total of more than 1100 hours operation has been logged under these conditions ${ }^{8}$. 


\section{THE X-FEL COUPLER}

Following the experience obtained to date, it has been decided to adopt the TTF-III coupler, with some minor modifications, as the coupler for the X-FEL project. The X-FEL linac will require 1000 couplers. The production of such a large number of couplers goes beyond all experience of such objects to date and will require the use of an industrial partner, or partners, in order to construct the couplers, with the required quality assurance, within a limited period of time ( $\sim 3$ years). The large number of examples implies an important attention to the unit cost of the coupler. At the time of writing we are in the process of starting industrial studies to investigate how best to manufacture the couplers such as to obtain economies of scale. One of the main driving factors in the cost of the proto-types has certainly been the required tolerances that are asked of the manufacturers. The dimensions of the coupler parts have been obtained from electro-magnetic simulations. However, the sensitivity of the RF performance to changes in these dimensions has been studied only recently. Simulations confirm that we have been over stringent on mechanical tolerances and that many of these can be greatly relaxed without compromising the performance. Following these calculations, we have prepared a complete set of engineering drawings, incorporating the new tolerances, so as to launch a call for tenders for studies of the industrialisation of, and the eventual production of, a large number $(500-1000)$ of TTF-III couplers. A computer aided design image of the X-FEL coupler is shown in figure 14. Two modifications, relative to Fig. 1 are evident. The electron pick-up ports are absent. For the X-FEL coupler it is foreseen to use the central antenna, already electrically isolated from the external diameter in order to apply the anti-multipactor DC bias, to 'sweep up' electrons emitted from whatever region of the coupler. This would allow one to reduce the number of diagnostic channels needed to protect the coupler (although one would lose all information regarding electron activity between the cold and warm parts). A second modification concerns the variation of the external Q. Until now, on TTF, the variation of $\mathrm{Q}_{\mathrm{ext}}$ is performed manually. However, for 1000 couplers, it is clear that the control of the $\mathrm{Q}_{\mathrm{ext}}$ must be performed remotely. For this reason an actuator is employed for adjustment of the antenna penetration in the beam pipe.

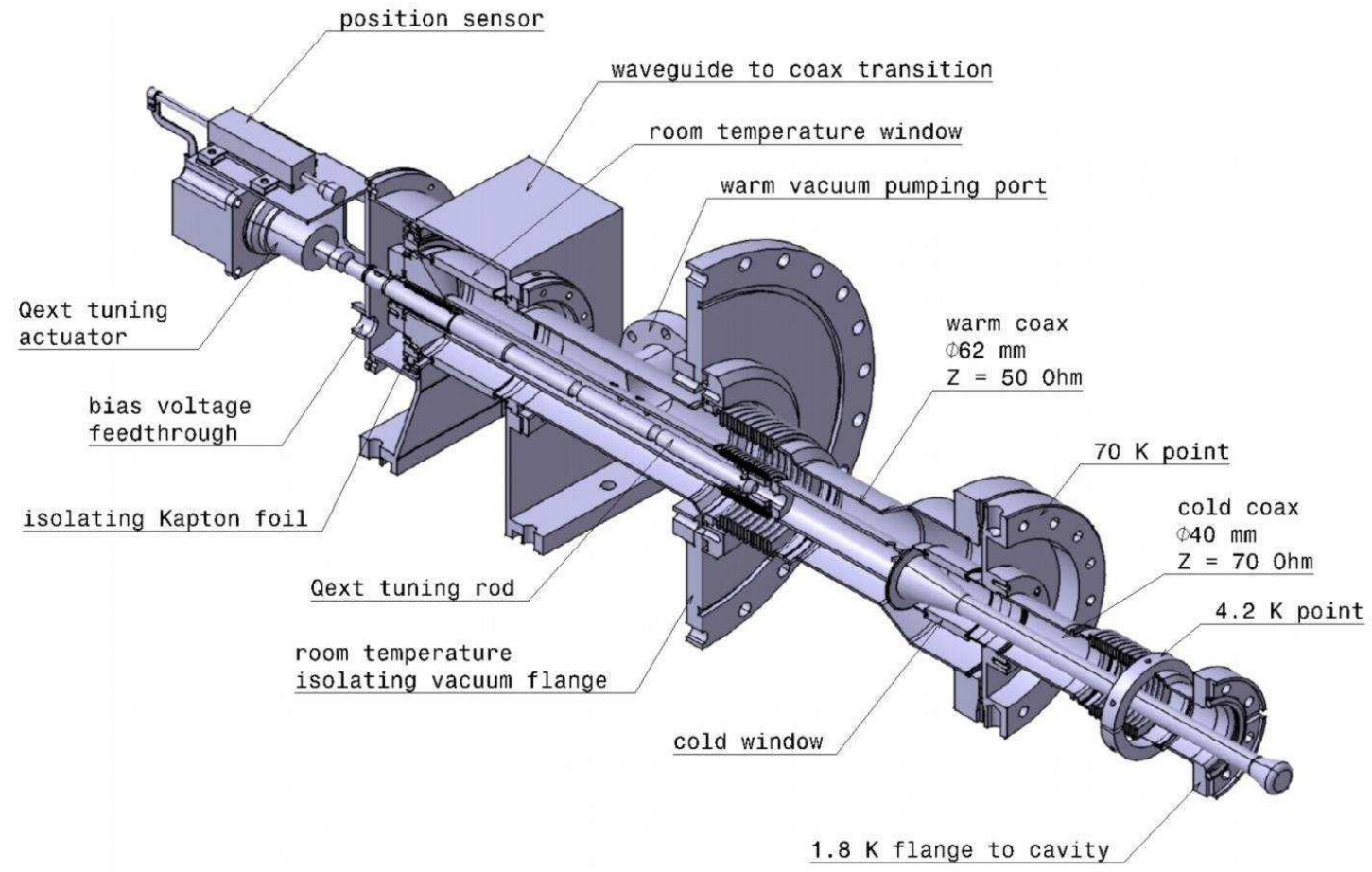

Figure 14. Computer Aided Design image of the TTF-III coupler modified for use with the X-FEL. 


\section{PROTO-TYPE COUPLER DESIGNS}

Although the TTF-III coupler meets the specifications for a nine cell TESLA cavity, the experience to date is that the unit cost of the coupler is higher than one would like. In addition, in the interest of reducing the number of required couplers and increasing the "fill factor" of the linac (defined as the active accelerating length to the overall linac length), proposals have been made for 'superstructure' cavities which would be composed of more than one (two or even four) multi-cell resonators powered by a single coupler. Here we describe three different proto-types which have been studied in view of such a scenario. One should note that these couplers were studied in the context of the $800 \mathrm{GeV}$ up-grade version of the TESLA proposal. Nevertheless, they may be of interest for the TeV collider currently under study by the ILC collaboration.

\subsection{The TTF-IV coupler}

The proposal for a "superstructure" composed of four cavities, each of 7 cells, for an up-graded $800 \mathrm{GeV}$ version of TESLA would require the use of a coupler capable of transmitting $2 \mathrm{MW}$ of power ${ }^{10}$. The RF design of such a prototype 'TTF-IV' coupler has been studied at DESY and the mechanical conception was developed at Orsay (Fig. 15).

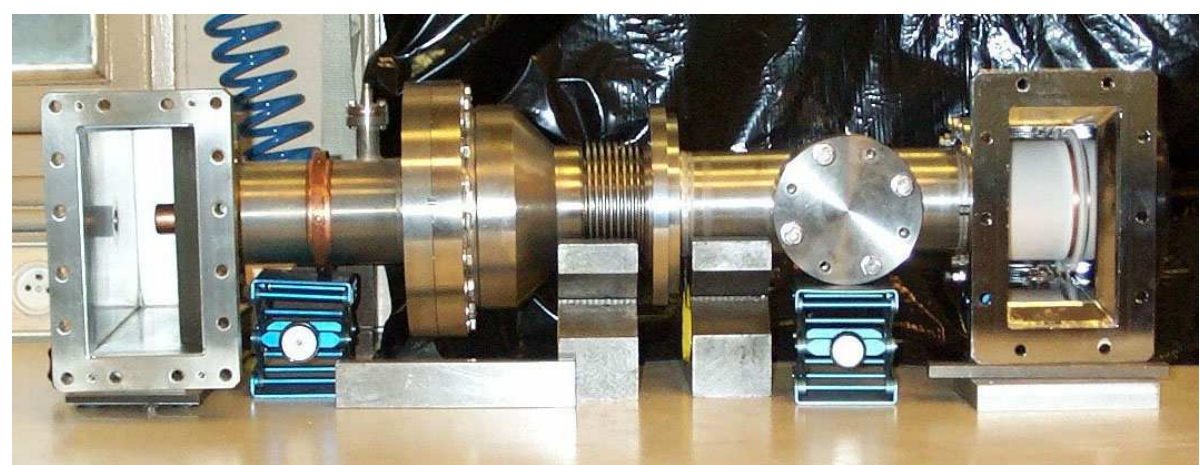

Figure 15. The TTF-IV coupler. The antenna can be seen protruding into a wave-guide to the left. The warm window within the transition is visible to the right.

This coupler design is based on the two-window geometry of TTF-III. However, in the interest of pushing multipactor levels to higher powers, the o.d. has been increased to $80 \mathrm{~mm}$. The insulation for the DC bias is provided by a ceramic disc placed in a high-voltage feed-through box over the wave-guide transition. Five such couplers have been manufactured to date. Electrical breakdown has been observed at $190 \mathrm{~kW}$ operation in the air side of the warm window. Further tests on these proto-types are awaiting completion of the conditioning of the CPI couplers. One disadvantage of such a large coupler diameter is the extent of the RF "kick" imparted to the electron beam. As the couplers are mounted on one side of the cavity, they break the cylindrical symmetry of the structure and introduce a transverse kick to the beam. The extent of the kick given to each electron as it passes the coupler depends on the longitudinal position of the particle within the bunch. The different perturbations can result in an increase of the emittance of the beam, which would reduce the luminosity of the collider. The overall magnitude of this effect would need to be evaluated before proposing the TTF-IV coupler for use with a linear collider.

\subsection{The TTF-V Coupler}

The possibility of a $2 \times 9$ cell cavity has also been proposed as a structure for TESLA. For this case we have studied the use of a coupler with an outer diameter of intermediate value to that of TTF-III and TTF-IV. The warm part of the coupler is essentially TTF-III. However, the cold part has been scaled up to the same outer diameter of $62 \mathrm{~mm}$ and has an impedance of $50 \Omega$. 

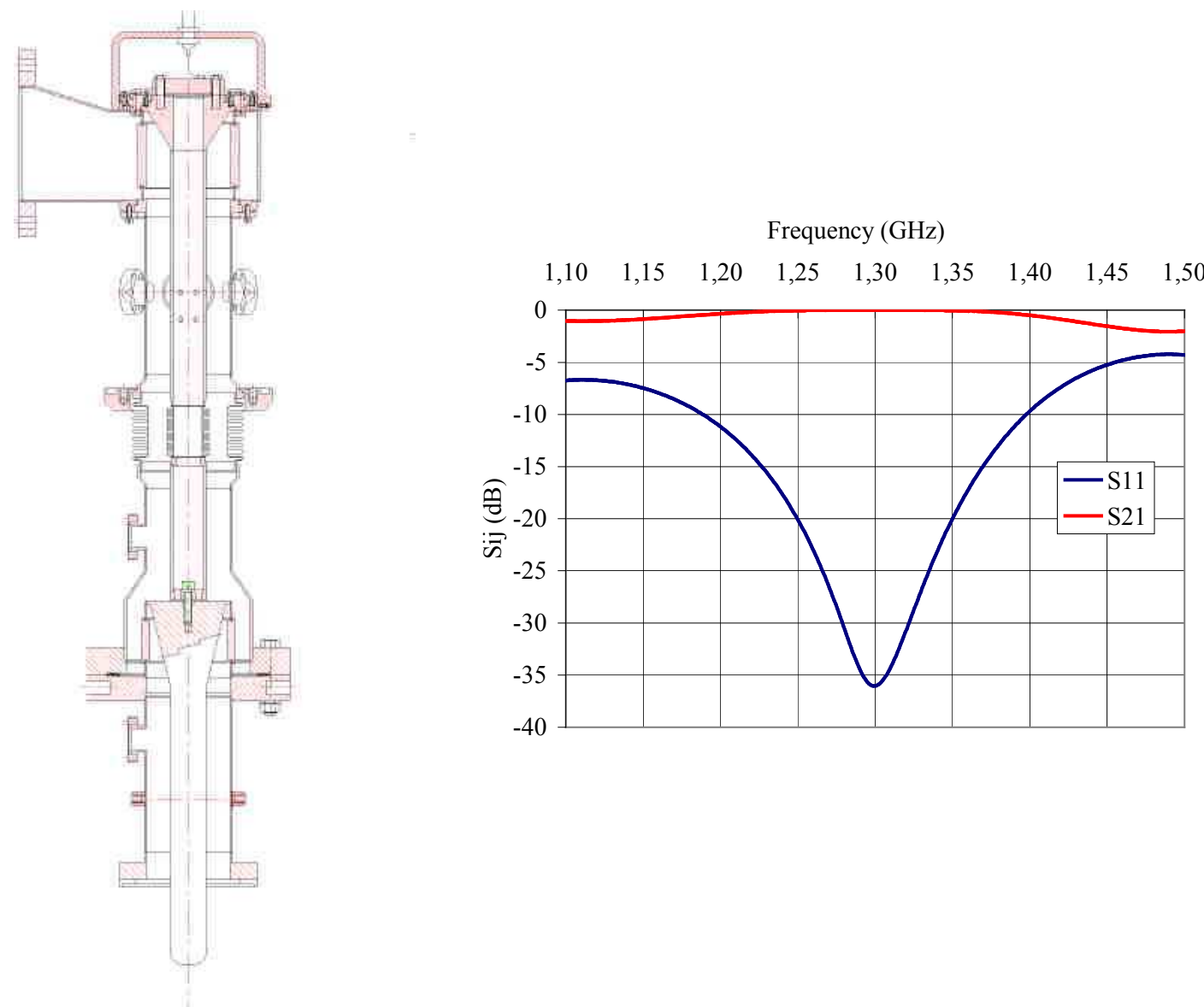

Figure 16. Left: Schematic of TTF-V coupler: Right: Calculated insertion and return loss using HFSS.

\subsection{The TW60 coupler}

The TW60 coupler (TW60 for Traveling Wave $-60 \mathrm{~mm}$ outside diameter) is a radically different design from the series of TTF couplers. The ceramic windows are of the thin disk $(8 \mathrm{~mm})$ type. This choice of ceramic was made on the basis that brazing them into the co-axial line should be technically simple and, thus, relatively inexpensive. The main interest therefore is the hope of building a cost effective coupler capable of sustaining the required RF power. Such windows also have the advantage that their RF surfaces are tangential to the plane of the TEM wave propagating in the co-axial line. Therefore they should, in principle, be insensitive to multipactor. Disk windows, however, are not necessarily well matched from an RF point of view as the dielectric constant of the window alters the impedance of the co-axial line. Matching would require a window of thickness equal to a half-wavelength (in the dielectric material). However, a window of this thickness would increase the dielectric losses beyond an acceptable level. In order to provide an overall matching of the wave-guide transition and the warm window, we employ a two-step, reduced height wave-guide transition. The reflection due to this WG cross-section change is cancelled out by the reflection due to the disk thus matching the transition. The disadvantage of this solution is the presence of a standing wave between the WG step and the warm ceramic. However the calculated fields are such that RF breakdown should not be an issue. The matching of the cold window is obtained differently. The outer diameter of the co-ax is reduced a short distance up and downstream of the ceramic. This is an inductive reactance to compensate the capacitive impedance introduced by the disk. Thus the line is matched to the co-ax, again at the expense of a standing wave component. This "traveling wave" approach to matching couplers has been proposed elsewhere ${ }^{11}$. Low power measurements of a TW60 coupler have already been performed $^{12}$.

Four couplers of each type, TW60 and TTF-V, have been ordered from the company ACCEL (Germany). They will be delivered early in 2006 and for high power tests. 


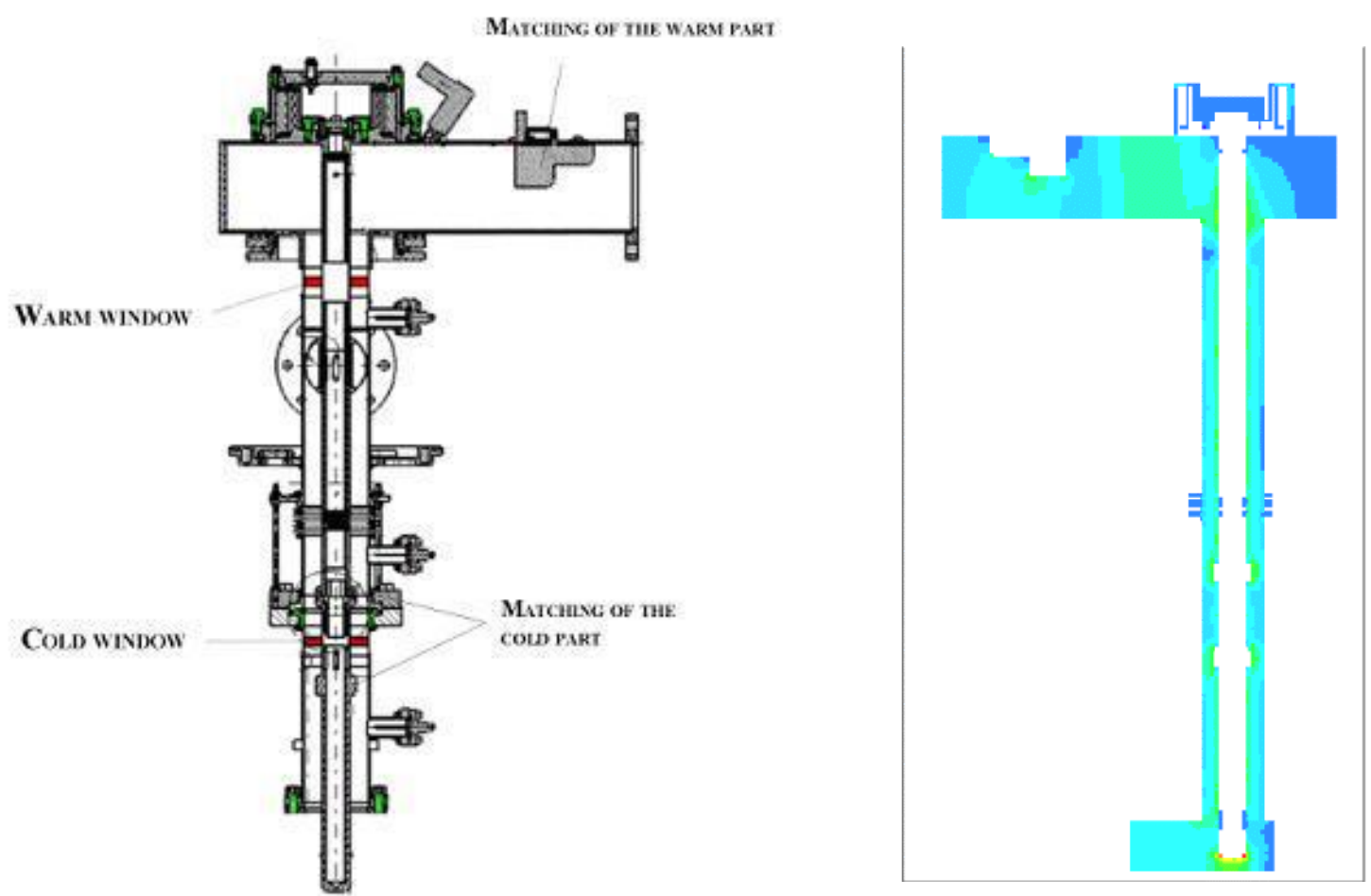

Figure 17. Left: Drawing of the TW60 coupler; Right: Electro-magnetic simulation of TW60 coupler fields.

\section{CONCLUSIONS}

Tests at DESY and Orsay have shown that the TTF-III coupler can comfortably sustain $1 \mathrm{MW}$ power levels. These couplers have now been adopted for use with the future European X-ray FEL. In principle they are also excellent candidates for use with a $1 \mathrm{TeV}$ linear collider (ILC). In view of the large number of couplers needed for the X-FEL ( $1,000)$ and for ILC $(\sim 20,000)$ there is a strong desire to reduce the conditioning time presently measured with these couplers ( $\sim 40-70$ hours). A program of R\&D is in progress at LAL-Orsay to understand the limitations behind the conditioning time and to find new 'recipes' for faster conditioning. The large number of couplers needed for these projects imply also that serious reductions in the unit cost should be aimed for. A joint DESY/LAL venture on industrialization studies is planned to achieve this reduction. In parallel with the effort on TTF-III couplers, alternative proto-types are being investigated in the hope that they may lead to some degree of performance and/or cost advantage for the ILC.

\section{ACKNOWLEDGEMENTS}

The work described in this paper represents the efforts of numerous physicists, engineers and technicians from LAL and DESY. In particular, the authors acknowledge the contributions of F. Barjot, S. Cavalier, T. Chabaud, F. Cordillot, M. Courtois, O. Dalifard, A. Gonnin, L. Grandsire, H. Jenhani, P. Lepercq, B. Mercier, M. Omeich, S. Prat, R. Panvier, C. Prevost, V. Thiebault, A. Variola and J. Vieira (LAL); S. Choroba, B. Dwersteg, D. Kostin, A. Labanc, C. Martens, A. Matheisen and D. Proch (DESY). The creation of the DESY-LAL coupler collaboration was largely due to the initiative of D. Trines (DESY) and F. Richard (LAL). 


\section{REFERENCES}

1. B. Aune et. al., The Superconducting TESLA Cavities, DESY Pre-print DESY 00-031, 2000.

2. R. Brinkmann, K. Flöttmann, J. Rossbach, P. Schmüser, N. Walker and H. Weise (Eds.), TESLA Technical Design Report, Part II The Accelerator, DESY Report 2001-011, 2001.

3. M. Champion, Design, Performance and Production of the FERMILAB TESLA RF Input Couplers, Proceedings of the $18^{\text {th }}$ International Linear Accelerator Conference, pp 521-524, (Geneva)1996.

4. W.-D. Moeller, High Power Coupler for the TESLA Test Facility, $9^{\text {th }}$ Workshop on RF Superconductivity, (Santa Fe, NM), Los Alamos Report LA-13782-C, 1999.

5. B. Dwertsteg, D. Kostin, M. Lalayan, C. Martens and W.-D. Möller, TESLA RF power Couplers Development at DESY, 10th Workshop on RF Superconductivity, pp 443-447, (Tsukuba, Japan) 2001.

6. Kostin and W.-D. Möller, Status and Operating Experience of the TTF Coupler, Proceedings of the $22^{\text {nd }}$ International Linear Accelerator Conference, pp 156-158, (Lübeck) 2004.

7. See for example, T.P. Wangler, RF Linear Accelerators, Chapter 5, John Wiley and Sons, ISBN 0-471-16814-9.

8. J. Lorkiewicz et. al., Characteristics of TiN Anti-multipactor Layers Reached by Titanium Vapour Deposition on Alumina Coupler Windows, Proceedings of the $11^{\text {th }}$ Workshop on RF Superconductivity

(Lubeck/Travemünde), 2003.

9. H. Jenhani, Conditionnemnt des coupleurs de puissance HF pour cavités supraconductrices en mode pulsé, Journées Jeunes Chercheurs, (Ile de Berder, France), http://events.lal.in2p3.conferences/JJC04/, 2004.

10. J. Sekutowicz, M. Ferrario and Ch. Tang, Superconducting superstructure for the TESLA collider: A concept, Physical Review Special Topics: Accelerators and Beams, Vol. 2 062001-7, 1999.

11. S. Yu. Kazakov, Increased Power RF Window, BINP Preprint 92-2, Protvino, 1992.

12. P. Lepercq, L. Grandsire, R. Panvier and T. Garvey, Design and Low Power Tests of an Input Coupler, High Power Coupler Workshop (Virginia), www.jlab.org/HPC2002, 2002. 